$\stackrel{N / 2}{N}$

Global Journals Inc

(2)

\title{
Dynamic Strategic and Organizational Capabilities
}

By Julian Miranda Torrez

Abstract- In the reviewed literature, dispersion was identified in the concepts used in the definition of dynamic capacities (CD), incomplete theories were also observed on which the proposed concepts were based, which has hindered the development of theoretical knowledge and the conduct of research empirical. The effort of the authors to solve this problem has been partial since it was analyzed from the perspective of strategy theory, the theory of organizations and administration (TOA) was not considered, this situation affected the clarity and precision of the CD.

The objective was raised, to build the concept of dynamic strategic and organizational capacities (CDEO) based on the theories of strategy (TE), organizations (TO) and administration (TA), in order to modernize the traditional concept dynamic capabilities (CD) and provide a solution to the concept dispersion problem.

Originality. Integration of theories around the CDEO construct, which will allow the theoretical and empirical development.

Keywords: dynamic strategic, dynamic capabilities, organizations, environment, competitive advantage, organizational performance.

GJMBR-A Classification: JEL Code: M10

Strictly as per the compliance and regulations of:

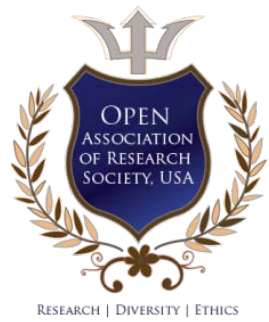

(c) 2021. Julian Miranda Torrez. This is a research/review paper, distributed under the terms of the Creative Commons AttributionNoncommercial 3.0 Unported License http://creativecommons.org/licenses/by-nc/3.0/), permitting all non commercial use, distribution, and reproduction in any medium, provided the original work is properly cited. 


\title{
Dynamic Strategic and Organizational Capabilities
}

\author{
Las capacidades Dinámicas Estratégicas y Organizacionales
}

\author{
Julian Miranda Torrez
}

\begin{abstract}
In the reviewed literature, dispersion was identified in the concepts used in the definition of dynamic capacities (CD), incomplete theories were also observed on which the proposed concepts were based, which has hindered the development of theoretical knowledge and the conduct of research empirical. The effort of the authors to solve this problem has been partial since it was analyzed from the perspective of strategy theory, the theory of organizations and administration (TOA) was not considered, this situation affected the clarity and precision of the CD.

The objective was raised, to build the concept of dynamic strategic and organizational capacities (CDEO) based on the theories of strategy (TE), organizations (TO) and administration (TA), in order to modernize the traditional concept dynamic capabilities (CD) and provide a solution to the concept dispersion problem.

Originality. Integration of theories around the CDEO construct, which will allow the theoretical and empirical development.
\end{abstract}

Абстрактный- En la literatura revisada se identificó dispersión en los conceptos utilizados en la definición de las capacidades dinámicas (CD), también se observó teorías incompletas en la que se basaron los conceptos propuestos, lo cual ha dificultado el desarrollo del conocimiento teórico y la realización de investigaciones empíricas. El esfuerzo intelectual de los autores por solucionar este problema ha sido parcial ya que se analizó desde la perspectiva de la teoría de la estrategia, no se consideró la teoría de las organizaciones y de la administración (TOA), esta situación afectó en la claridad y precisión del constructo $C D$.

Se planteó el objetivo principal, construir el concepto de capacidades dinámicas estratégicas y organizacionales (CDEO) fundamentado en las teorías de la estrategia (TE), de las organizaciones (TO) y de la administración (TA), a fin de modernizar el concepto tradicional de las capacidades dinámicas (CD) y aportar una solución al problema de dispersión de conceptos.

Originalidad. Integración de teorías y diferentes perspectivas en torno al constructo CDEO, lo cual permitirá el desarrollo teórico y empírico sobre el tema.

Keywords: dynamic strategic, dynamic capabilities, organizations, environment, competitive advantage, organizational performance.

Author: e-mail: julianmt4@gmail.com

\section{INTRODUCTION}

a perspectiva de las CD actualmente es un tema central en la administración estratégica, tiene su influencia en la investigación teórica de las estrategias, ventaja competitiva, desempeño, creación de valor en las organizaciones, entre otros temas. A pesar de su importancia adquirida, el constructo está en proceso de construcción teórica y empírica, se presenta una dispersión en los conceptos como se muestra en el cuadro 1, en las definiciones de este constructo por ejemplo se utilizaron los siguientes términos: habilidad (Teece, Pisano y Shuen (1997), competencias (Helfat, 1997), capacidad (Collins, 1994), procesos (Vivas, 2005), lo cual ha creado varias inconsistencias en el uso del concepto (Pavlou y Sawy, 2011), por lo que ha sido criticado (Kuuluvainen, 2012); esta situación dificultó en la identificación de sus dimensiones y variables que permitan realizar investigaciones empíricas (Ambrosini y Bowman, 2009).

Una de las causas de esta problemática es debido a que no se plantea con precisión las teorías que en las que se fundamenta el concepto de CD. Teece, Pisano, y Shuen (1997) son los autores precursores del concepto, en su planteamiento parcialmente toman en cuenta la TE y no consideraron la teoría de la capacidad de la organización, tampoco las teorías de la organización y de la administración como fundamentos teóricos de las CD. Teece (2017, 2014) se propone construir una teoría de las capacidades de la empresa desde las perspectivas de la teoría de la estrategia y de la economía, para redefinir el concepto planteado en 1997; en esta propuesta ya se observa la intensión de incorporar conceptos de la teoría de las organizaciones y de la administración en el constructo. El objetivo de esta investigación ha sido el de contribuir en la construcción teórica, por lo que se ha propuesto conceptualizar el constructo CDEO fundamentado en las TE y TOA y crear una perspectiva integradora y multidimensional.

Con el objetivo de lograr el objetivo propuesto, las preguntas que guiaron las investigaciones fueron: ¿qué teorías se pueden utilizar para construir una nueva conceptualización de este constructo con una perspectiva estratégica y organizacional? ¿Qué factores contingentes inciden en la planeación e 
implementación del constructo? ¿Qué nuevos temas de investigación se proponen para profundizar en el estudio teórico y realizar investigaciones empíricas?

El documento se desarrollará en los siguientes apartados. En la primera parte, presentaremos una síntesis de las teorías en la que se fundamenta la conceptualización del constructo CDEO. En la segunda parte plantearemos la conceptualización del constructo sustentada en las teorías de la estrategia, de las organizaciones y de la administración. En la tercera parte analizaremos los factores contingentes que afectan al constructo. Finalizaremos el documento presentando las conclusiones y los temas de investigación que se puedan estudiar en el futuro.

\section{il. Methodology}

La metodología utilizada para la revisión de la literatura siguió una versión simplificada del proceso descrito por Tranfield, Denyer y Smart (2003). En primer lugar, se llevó a cabo una búsqueda simultanea de artículos publicados en la base de datos bibliográficos de Strategic Managememnt Society, Academy of Management, Cambridge University Press, Elsevier, Esmeralda, ABSCO, Oxford University Press y del Consejo Nacional de Ciencia y Tecnología, que contribuyeran con los temas dynamic capabilities, organizaciones, administración y estrategias. Luego se ordenaron los artículos en función del número de citas y mayor impacto, se seleccionaron aquellos de reciente publicación y resaltaran los aspectos teóricos y conceptualizaciones del constructo objeto de estudio. En la siguiente etapa, se leyeron y sintetizaron cada uno de los artículos, se utilizó mapas conceptuales que permitieran relacionar conceptos y documentos. En síntesis, se aplicó una metodología de análisis y critica documental.

\section{ili. Teorias Fundamentales}

El marco teórico de las CD tiende a reconciliar e integrar varias perspectivas y teorías, sin embargo, la mayoría de los teóricos analizaron este constructo desde la óptica de teoría de la estrategia, recientemente Teece (2017) analizó desde la perspectiva de la teoría economía, Winter (1917) en sus análisis de este constructo de manera incompleta incorpora conceptos de la TOA, Trau (2017) resalta los factores organizacionales y administrativos en las CD, pero no profundiza en sus planteamientos. Ritala, Heiman y Hurmelinna-Laukkamen (2016) resaltan que las organizaciones son un portafolio de capacidades, dinámicas y organizacionales. En los autores citados, se observa que existen diferentes teorías que sustentan las $C D$, sin embargo, no establecen relaciones claras entre estas teorías. Nuestro objetivo es llenar este vacío, por lo que a continuación presentaremos una descripción breve de algunas teorías directamente relacionadas con CDEO, en los anexos 1 y 2 se da un panorama general de las teorías.

\section{a) Teorías de las organizaciones y de la administración} (TOA)

Las TOA han sido poco referenciadas en el estudio de las $C D$, dieron mayor prioridad a la ya que, teoría de la estrategia, esta situación ha creado vacío en la información y problemas en la interpretación del concepto. Con el objetivo de solucionar esta situación en el anexo 1 se presenta un panorama amplio de las teorías, a continuación enfocaremos nuestra atención en las teorías de la contingencia y de las capacidades organizacionales al estar directamente relacionadas con CDEO.

\section{La teoría de la contingencia}

La teoría de la contingencia (Hatch, 1997; Lawrence y Lorsch, 1967), plantea la relación, la adaptación o el ajuste contingente del entorno con la organización (Volberda, Weerdt, Verwaall, Stienstra y Verdu, 2012), es decir, que la organización debe responder al dinamismo del entorno, implementando cambios estratégicos para sobrevivir y prosperar en un entorno contingente, como también, cambios que impulsen futuros desarrollos del entorno (Kim y Mauborgne, 1997). El concepto de ajuste también implica congruencia de los recursos, de las capacidades y las competencias de la organización con las condiciones cambiantes del entorno, es un alineamiento con las demandas externas (Kor y Mesco, 2012); el éxito de la organización depende del ajuste estratégico con su entorno, Rashidirad, Soltani y Syed (2013) resaltan el ajuste con la estrategia de la organización y los factores externos e internos.

El ajuste estratégico es un concepto central en la teoría de la contingencia, en la ecología poblacional, en la teoría de las organizaciones y en la administración estratégica (Volberda, Weerdt, Verwaall, Stienstra y Verdu, 2012). El concepto ajuste también implica congruencia del diseño organizacional (tecnología, estructura y cultura) y de las estrategias con las demandas del entorno, es decir, una congruencia de las variables del entorno con las variables internas de la organización, resaltándose las estrategias y las acciones como los factores claves para el asuste estratégico entre la organización y su entorno.

Por lo tanto, tomando en cuenta ambas teorías planteamos que las capacidades dinámicas más las estrategias apoyan a la organización a lograr un ajuste dinámico con el dinamismo del entorno, teniendo como resultados altos desempeños y ventaja competitiva sustentable (Rashidirad, Soltary y Salimian, 2014).

Teorías de las capacidades organizacionales (TCO)

Chandler fue el precursor en plantar la TCO en la administración, resaltó su estrecha relación con la estrategia, la estructura y el desempeño organizacional, esta teoría ha tenido una fuerte influencia en la teoría de 
la estrategia y TOA (Chandler, 1992). La capacidad organizacional (CO) es esencialmente un fenómeno social, potencialmente reside en los miembros de la organización (Ghoshal, Hahn y Moran, 1999); son aprendidas dentro de un contexto organizacional específico por lo que son difíciles de transferir de una organización a otra. Las capacidades aprendidas son unidades de análisis que permiten explicar el inicio y el crecimiento de las organizaciones modernas, los aprendizajes organizacionales permiten construir capacidades organizacionales.

Las capacidades organizacionales son: las habilidades, los conocimientos, las experiencias individuales y colectivas esenciales para explotar e intercambiar los recursos eficiente y efectivamente, permite la solución de problemas, toma de decisiones en los diferentes niveles de la estructura organizacional, la competencia administrativa, la exploración y explotación de las oportunidades en el mercado y responder a los cambios en el entorno (Chandler, 1992; Ghoshal, Hahn y Moran, 1999; Ritala, Heiman, Hurmelinnna-Laukkamen, 2016).

Las organizaciones incluyen un conjunto amplio de capacidades, habilidades, aprendizajes y experiencias centradas en el factor humano, como también, rutinas y procesos en el uso y transformación de los recursos; estos conceptos están estrechamente relacionados. Las capacidades organizacionales son más que la suma de sus partes (Chandler, 1992), su aplicabilidad está limitada por los directivos, administradores y recursos humanos ubicados en la estructura organizacional. Estos planteamientos tienen sus fundamentos en la teoría basada en los recursos y en la teoría de la contingencia (Andrews, Beynon y McDemott, 2016).

Las capacidades organizacionales están relacionadas con los conceptos estructura organizacional y con la estrategia. Las capacidades se pueden ubicar en los niveles superiores, intermedio y operativo; en la cúspide de la organización tienen características estratégicas al abarcar toda la organización y mantener una interrelación con el entorno; en el nivel intermedio se refieren a las capacidades funcionales (mercadotecnia, finanzas, recursos humanos y producción), y en los niveles bajos de la estructura tienen características de capacidades operativas. Las capacidades dinámicas se relacionan con las capacidades ubicados en la cúspide de la organización y son estratégicas al responder a los movimientos de los competidores y de lograr un ajuste estratégico con los cambios constantes en la economía, en el entorno social y político. La estrategia está relacionada con las capacidades, define el como la organización responderá a los cambios del entorno, satisfacer las expectativas de los clientes, responder a la competencia, su esencia es crear y usar la ventaja competitiva (Chandler, 1992).
Teorías de la estratégica (TE)

Chandler (1992) entre otros autores plantearon los fundamentos teóricos de la TE, analizó las relaciones entre estrategia y la estructura organizacional, la teoría de las capacidades permite relacionar la TE y TOA. Las perspectivas basadas en los recursos y el conocimiento son los antecedentes teóricos inmediatos de las CD.

\section{La perspectiva basada en los recursos}

(Barney, 1997; Denford, 2013; Teece, 2014) se origina en los trabajos de Perose (1959). Gupta (2014) plantea que las organizaciones están integradas por recursos y capacidades. Los recursos son definidos como el conjunto de conocimientos, activos físicos, capital humano, y otros factores tangibles e intangibles que las organizaciones poseen y controlan para producir productos y servicios con los objetivos de lograr ventaja competitiva en el largo plazo, crear valor para los participantes en la organización y obtener altos desempeños utilizando diversos recursos, resaltándose las características de ser evaluables, raros, difíciles de imitar y no sustituibles, disponibles en la organización y en el entorno (Peteraf, 1993; Gupta, 2014). Esta teoría es estática, por lo que se propone como una extensión la perspectiva de las capacidades dinámicas, resaltando que las características de los recursos cambian por el dinamismo del entorno (Ambrosini y Bowman, 2009). En el anexo 2 se presentan otras teorías de la estrategia.

Perspectiva tradicional de las capacidades dinámicas

El concepto de CD inicialmente fue propuesto por Teece, Pisano y Shuen (1997), posteriormente ha sido estudiado por diferentes autores. Barreto (2010) se enfocó en definir y clasificar la amplitud del concepto, Ambrosini y Bowman (2009) analizaron el desarrollo histórico del concepto e identificaron algunos de sus supuestos fundamentales, DiStefano, Peteraf y Verona (2010) realizaron un análisis de co-citación para explorar la estructura del dominio de la investigación de este tema a fin de lograr un mejor entendimiento de sus orígenes del concepto, estado actual y desarrollo futuro por medio de la investigación, Peteraf, Stefano y Verona (2013) utilizaron la técnica de medición bibliográfica para analizar a los autores más citados sobre el concepto (Vogel y Guttel, 2013). La aportación de estos autores al desarrollo del concepto fue parcial, ya que no proponen una definición integral, utilizaron diferentes términos y enfoques teóricos para definir este constructo, como se muestra en la tabla 1. 
Tabla 1: Definiciones de capacidades dinámicas (CD)

\begin{tabular}{|c|c|}
\hline Autores & Definiciones de capacidades dinámicas \\
\hline $\begin{array}{l}\text { Teece, Pisano y } \\
\text { Shuen (1997) }\end{array}$ & $\begin{array}{l}\text { La habilidad de la firma para integrar, construir y reconfigurar competencias internas } \\
\text { y externas en función de cambios rápidos en el entorno. }\end{array}$ \\
\hline Helfat (1997) & $\begin{array}{c}\text { El subconjunto de competencias /capacidades, las cuales permiten a la firma crear } \\
\text { nuevos productos y procesos, y responder a las circunstancias de mercado } \\
\text { cambiantes. }\end{array}$ \\
\hline Collins (1994) & La capacidad para desarrollar e innovar más rápidamente. \\
\hline Vivas (2005) & $\begin{array}{c}\text { Las capacidades dinámicas son complejos procesos organizacionales de alto nivel, } \\
\text { las cuales proporcionan las condiciones adecuadas para la modificación y } \\
\text { renovación de los activos de la organización }\end{array}$ \\
\hline $\begin{array}{l}\text { Eisenhard y Martin } \\
\qquad(2000)\end{array}$ & $\begin{array}{c}\text { Los procesos de la firma que utilizan recursos -específicamente los procesos para } \\
\text { integrar, reconfiguran, obtener y liberar recursos- para ajustarse a aun crear cambios } \\
\text { en el mercado. Las CD son las rutinas organizacionales y estratégicas por las cuales } \\
\text { las firmas logran nuevas configuraciones de recursos mientras el mercado emerge, } \\
\text { colisiona, se divide, evoluciona o muere. }\end{array}$ \\
\hline Zahra y George (2002) & $\begin{array}{c}\text { Capacidades orientadas al cambio, que ayudan a las organizaciones reutilizar y } \\
\text { reconfigurar sus bases de recursos para cumplir con las demandas de los clientes y } \\
\text { enfrentar las estrategias de la competencia. }\end{array}$ \\
\hline Winter (2003) & $\begin{array}{c}\text { Capacidades que operan para extender, modificar o crear las capacidades } \\
\text { ordinarias o sustantivas. }\end{array}$ \\
\hline Teece (2017) & $\begin{array}{c}\text { Las CD son competencias de alto nivel que determinan la habilidad de una firma } \\
\text { para integrar, construir y reconfigurar competencias/recursos internos y externos } \\
\text { para enfrentar entornos de negocios de alto dinamismo. Ellas determinan la } \\
\text { velocidad y el alcance con que los recursos de la firma pueden ser alineados y } \\
\text { realineados para ajustarse a las oportunidades del entorno de negocios así como } \\
\text { generar rentas sostenibles extraordinarias. Las CD pueden ser útiles para tres tipos } \\
\text { de actividades: 1) identificación y evaluación } \\
\text { de una oportunidad (sensing); 2) movilización de recursos para aprovechar una } \\
\text { oportunidad y capturar valor al hacerlo (seizing); y 3) renovarse continuamente } \\
\text { (transforming). }\end{array}$ \\
\hline
\end{tabular}

Fuente: elaboración propia

En la tabla 1 se observa que los autores utilizaron diferentes términos para conceptualizar CD, por ejemplo: habilidad (Teece, Pisano y Shuen (1997), competencias (Helfat, 1997), capacidad (Collins, 1994), procesos (Vivas, 2005), lo cual ha creado varias inconsistencias en el uso del concepto CD (Pavlou y Sawy, 2011), por lo que ha sido criticado (Kuuluvainen, 2012); las propuestas de conceptos dispersaron su significado elevando el nivel de abstracción y complejidad del constructo (Dierickx y Cool, 1989), esta situación dificultó en la identificación de sus dimensiones y variables en la realización de investigaciones empíricas (Ambrosini y Bowman, 2009).

Refiriéndonos de manera más específica a la definición de CD propuesto por Teece, Pisano y Shuen (1997), se observan que los autores solo se refieren a las competencias internas y externas, no toman en cuenta otros aspectos de la organización como la cultura, la estructura, toma de deciones, modelo de negocio. En esta definición hay ausencia de los conceptos administrativos.

b) Construcción del concepto de las capacidades dinámicas estratégicas y organizacionales

Los planteamientos tradicionales de las CD han sido abordados a partir de la teoría de la estratégica y no se tomó en cuenta las TOA, esta situación tiende a cambiar como ejemplos se presentan los siguientes avances: Easterby-Smith y Prieto (2008) relaciona las CD con la administración del conocimiento, Teece (2017) realiza una revisión crítica de la teoría de la firma (organización) y de la teoría neoclásica de la economía como antecedentes de la teoría de la capacidad, plantea que las empresas son organizaciones con capacidades y estrategias, Manning, Hutzschenreuter y Strathmann (2012) analizaron la relación de la administración con las CD, resaltaron que la interrelación de las habilidades individuales y las rutinas organizacionales requieren la interface de la administración, es decir, de las actividades de comunicación, operación, procesos, financieros y de mercadotecnia. En el planteamiento clásico de las CD estas actividades administrativas son secundarias y en muchos casos no son tomados en cuenta.

Las teorías de las organizaciones y de la administración (TOA) permiten explicar desde una perspectiva amplia y multidimensional el funcionamiento y desarrollo de las organizaciones, así como, sus relaciones con el entorno. Las organizaciones incluyen un conjunto amplio de capacidades, rutinas, procesos, toma de decisiones (Simón, 1979) entre otros temas, 
los cuales son agrupados en el constructo capacidades organizacionales (CO).

La teoría de la estrategia (TE) incluye una temática muy amplia, algunos temas son presentados en el anexo 2 relacionadas con la perspectiva de las CD. Estos planteamientos teóricos son agrupados en el constructo estrategia.

La interrelación de las teorías TOA con la TE permiten plantear una nueva perspectiva integral de las CDEO, es decir que este constructo es hibrido al estar relacionado con un amplio conjunto de teorías. Esta propuesta es un aporte significativo en la construcción de la teoría de las capacidades dinámicas, permite fortalecer los planteamientos de Teece (2017), y solucionar el problema de la dispersión de los términos utilizados en la conceptualización de este constructo.

Tomando en cuenta los insumos teóricos que anteceden planteamos la conceptualización de CDEO: son las habilidades individuales y colectivas de utilizar, crear, transformar recursos y decidir implementar las estrategias y las capacidades organizacionales para responder rápidamente al dinamismo del entorno y a las necesidades de la organización, a fin de mantener la ventaja competitiva, e incrementar constantemente el desempeño organizacional. Esta conceptualización se sustenta en los autores como se muestra en metacuadro 2, los insumos teóricos se presentan en los anexos 1 (TOA) y 2 (TE) y en los cuadros 1,2 , 3, y 4 .

Tabla 2: Fundamentación teórica de CDEO

\begin{tabular}{|c|c|}
\hline Conceptos utilizados en CDEO & Autores en las que se sustenta \\
\hline Organización & March y Simón (1980); Augier y Teece (2006); Winter (2017); Trau (2017) \\
\hline Habilidades & Cuadro 1, Barnard (1938) \\
\hline Individuales y colectivas & Helfat y Peteraf (2015); Helfat y Peteraf (2015); Grupta (2014); Gary y Wood (2011). \\
\hline $\begin{array}{l}\text { Utilizar, crear y transformar } \\
\text { (mas otras estrategias) }\end{array}$ & Tabla 3 \\
\hline $\begin{array}{l}\text { Recursos (y otros aspectos } \\
\text { como las competencias, } \\
\text { capacidades, conocimientos, } \\
\text { modelos de negocios, activos) }\end{array}$ & $\begin{array}{c}\text { Barney (1997); Denford (2013); Teece (2014); Grant (1996); Braganza, Brooks, } \\
\text { Nepelski, Ali y Moro (2017); Zheng, Zhang, Wu y Du (2011 }\end{array}$ \\
\hline Decidir implementar & Luhmann (2012); Simón (1979); Barnard (1938) \\
\hline Las estrategias & Cuadro 3; Mintzberg (1989); Chandler (1992); \\
\hline Capacidades organizacionales & $\begin{array}{c}\text { Chandler (1992); Tseng y Lee (2014); Tzortzki y Mihiotis (2014); Ritala, Heiman, } \\
\text { Hurmelinnna-Laukkamen (2016); Ghoshal, Hahn y Moran (1999); Andrews, Beynon } \\
\text { y McDemott (2016); Ulrich y Lake (1991); Teece }(2017,2014)\end{array}$ \\
\hline Responder rápidamente & Ambrosini y Bowman (2009); \\
\hline Dinamismo del entorno & Wang (2016); Shara y Vredenburg (1998); Gupta (2014); \\
\hline $\begin{array}{l}\text { Necesidades de la } \\
\text { organización }\end{array}$ & $\begin{array}{c}\text { Rashidirad, Soltani y Salimian (2014); Foss, Lyngsie y Zahra, (2013); Fainshmidt y } \\
\text { Frazier (2016); Gupta (2014); Mintzberg (1989) }\end{array}$ \\
\hline Ventaja competitiva & (Schilke, 2014); Porter (1987); Rashidirad, Soltani y Syed (2013). \\
\hline Desempeño organizacional & Laaksone y Peltoniemi (2016); Miranda, 2014) \\
\hline
\end{tabular}

Fuente: Elaboración propia

En el concepto de CDEO propuesto resalta los siguientes aspectos:

- Permite unificar los conceptos que dispersaron la naturaleza de las CD.

- Contribuye en unificar las TOA con TE, campos del conocimiento que se han desarrollado en forma paralela, por lo tanto, esta perspectiva permite ampliar las relaciones de las comunidades académicas e interactuar en la discusión sobre el constructo.

- Facilita el desarrollo de nuevos conocimientos teóricos y realizar investigaciones con mayor impacto con una perspectiva multidimensional y multifactorial.

En los planteamientos tradicionales de las CD parcialmente tomaron en cuenta las estrategias como respuesta al dinamismo del entorno. En el concepto estrategias se incluyen las acciones, actividades y los procesos planeados e implementados por los directivos que ocupan puestos en la alta gerencia, o las funciones que realizan los ejecutivos para responder al dinamismo del entorno y cumplir con las necesidades de la organización, con los objetivos de mantener una ventaja competitiva e incrementar constantemente el desempeño organización en cumplimiento de objetivos estratégicos. En la tabla 3 se presenta ejemplos de estrategias que apoyan la realización de las CDEO. 
Tabla 3: Estrategias

\begin{tabular}{|c|c|}
\hline Estrategias & Autores \\
\hline Integrar & Teece, Pisano y Shuen (1997); Denford (2013); \\
\hline Reconstruir & Teece, Pisano y Shuen (1997); \\
\hline Reconfigurar & Zahra y George (2002); Denford (2013) \\
\hline Crear & Helfat (1997); Denford (2013) \\
\hline Desarrollar & Collins (1994); Denford (2013); \\
\hline Innovar & Standler, Helfat y Verona (2013) \\
\hline Modificar, renovar, extender & Cohen y Levintahl (1990) \\
\hline Absorber conocimiento del entorno & Grimaldi, Quinto y Rippa (2013); \\
\hline Buscar (sensing) y aprovechar & Kuuluvainen (2012); Enkel, Roseno y Mezger (2012); Pavlou y Sawy \\
(seizing) las oportunidades externas & (2011); Grimaldi, Quinto y Rippa (2013) \\
\hline Transformar la organización & Dixon, Meyer y Day (2010) \\
\hline Replicar & Denford (2013) \\
\hline Desarrollar (productos, procesos) & Schilke (2014) \\
\hline
\end{tabular}

Fuente: Elaboración propia

10 Niveles de dinamismo de las CDEO

En la definición propuesta se resalta el término dinámico, refiriéndose a la forma ágil en tiempo y forma de planear e implementar las estrategias, acciones y procesos más rápido que la competencia, es decir, es la agilidad de la organización en responder en forma proactiva o reactivamente al dinamismo del entorno (Shara y Vredenburg, 1998). Las CDEO proactivos modifican las características del entorno, de la industria y del mercado; y los reactivos son las respuestas que se realizan en las organizaciones de manera oportuna a los impactos del entorno a fin de lograr el ajuste estratégico.

El dinamismo se presentas en tres niveles: en el primer nivel, los cambios dinámicos se realizan en el entorno, en los mercados, en los sectores industriales, en la competencia, en los proveedores de insumos, servicios y productos; así como, cambios en los gobiernos y sistemas políticos regionales y a nivel global. El segundo nivel, corresponde a los cambios dinámicos que ocurren dentro de las organizaciones: son las modificaciones y reingeniería de los procesos, reconfiguración de los modelos de negocios, innovaciones dinámicas en la tecnología, productos y servicios. En el tercer nivel, el cambio dinámico se presenta en las relaciones de los entornos con las organizaciones.

Los tres niveles de dinamismo están estrechamente relacionados, interactúan siguiendo procesos no causales o de causalidad múltiple, con la organización en su relación con los entornos dinámicos y complejos.

Por lo tanto, el concepto de CDEO que se propone es multidimensional ya que incluye los tres niveles de dinamismo. Cada nivel es un sistema que incluye las interrelaciones multicausales no lineales entre sus variables formando modelos complejos.

Las CDEO pueden ser deliberadamente planeadas o surgir a través de procesos emergentes (Ambrosini y Bowman, 2009). Son deliberados cuando los entornos son estables, las estrategias, acciones y procesos son planeados e implementados siguiendo procesos formales y burocráticos; surgen CDEO emergentes cuando los entornos son altamente dinámicos y complejos, es decir, que las estrategias, acciones y procesos no son planeados de manera formal, son respuestas rápidas de la organización a las contingencias del entorno. Por lo tanto, las CDEO dependen de las características de los entornos y de la organización. El entorno se caracteriza por su dinamismo, complejidad e incertidumbre que crea nuevas oportunidades de negocios y presiones competitivas de los participantes externos (clientes, proveedores, nuevas organizaciones que entran a la industria). Las organizaciones se caracterizan por su arreglo organizacional (Mitzberg, 1989) y por su agilidad en la elección de las mejores estrategias acciones y procesos para dar respuesta al dinamismo y a las condiciones emergentes en los entornos.

\section{Factores contingentes que inciden las CDEO}

La planeación e implementación de las CDEO son afectados por el dinamismo del entorno, las características de las organizaciones, y por las capacidades cognitivas de los directivos y de los recursos humanos que participan en la organización. En el cuadro 4 se presentan los autores que la respaldan los factores contingentes.

\section{Factor 1: El dinamismo del entorno}

En la conceptualización de las capacidades dinámicas, el dinamismo del entorno es uno de los factores claves que impactan en el desarrollo de las organizaciones. En este tema, se incluye el dinamismo y la evolución de los mercados, cambios en la economía, surgimiento de nuevos segmentos de consumidores, la libre competencia, innovación de sistemas de producción y distribución de productos a nivel internacional. El cambio actual en la tecnología disminuye los ciclos de vida de los productos, lo cual se agiliza con la competencia global, y la rápida difusión de los conocimientos (Gupta, 2014). Los entornos 
dinámicos del mercado erosionan el valor del conocimiento organizacional y las capacidades centrales, lo cual incide en la utilización de los recursos, en la configuración de los modelos de negocios, en las capacidades y competencia de los participantes en la organización. Frente a esta situación los directivos de las organizaciones reconfiguran sus recursos, actualizan sus bases de conocimientos, implementan nuevas estrategias, acciones y procesos organizacionales para adaptarse al dinamismo del entorno, es decir, planean e implementan CDEO.

Factor 2: Las características de la organización

Las características de la organización es un tema muy amplio, incluye: la estructura y clima organizacional, el tamaño, la antigüedad, la edad, los procesos, y el modelo de negocios. Estructura de la autoridad y flujos de comunicación e información formal e informal dentro de la organización y con los participantes externos, estos aspectos inciden en la formación de las CD (Rashidirad, Soltani y Salimian, 2014). El diseño organizacional, centralización o descentralización, facilita significativamente el uso de los conocimientos en la innovación de los procesos de desarrollo de los portafolios de capacidades organizacionales, de productos y en las actividades de investigación y desarrollo, así como, en las decisiones de explotar las oportunidades estratégicas, así como, de planear e implementar capacidades dinámicas (Foss, Lyngsie y Zahra, 2013).

Factor 3: Las capacidades cognitivas de los recursos humanos

En la organización los recursos humanos son el factor principal en la implementación de las CDEO, los

Tabla 4: Factores contingentes en los constructos del modelo factores cognitivos y los modelos mentales de estos recursos tienen un impacto positivo o negativo en el funcionamiento de las organizaciones $y$ en la implementación de las capacidades. Helfat y Peteraf (2015) analizaron como la heterogeneidad de las capacidades cognitivas podrá producir heterogeneidad de las capacidades dinámicas entre los altos directivos, lo cual también incide en lograr diferentes desempeños organizacionales. Miranda (2014) plantea que los factores cognitivos (por ejemplo, memoria del pasado, percepciones acerca del presente, y expectativas del futuro), emotivos (por ejemplo, intereses y preferencias personales) y las relaciones entre las personas afectan al desarrollo de las capacidades dinámicas, a las decisiones estratégicas efectivas, a la ventaja competitiva sustentable y a la efectividad organizacional. Gary y Wood (2011) plantean que los modelos mentales administrativos son determinantes críticos de la elección y decisiones de implementar estrategias (Simón, 1979), ya que son estructuras de conocimientos simplificados o representaciones cognitivas acerca del entorno y de las áreas de trabajo de los negocios. Los directivos de las organizaciones tienen modelos mentales que incluye la organización y el dinamismo del entorno que incide en el desempeño. Las diferencias en modelos mentales ayudan a explicar por qué los administradores adoptan diferentes estrategias y logran diferentes niveles de éxito. En el tabla 4 se sintetizas estos factores.

Fuente: Elaboración propia

\section{Conclusion}

Se dio el primer paso en la construcción teórica de las CDEO, es una alternativa para solucionar la dispersión de los términos utilizados para definir el concepto tradicional de las capacidades dinámicas inicialmente propuesta por Teece, Pisano y Shuen (1997), creador de esta perspectiva. En esta misma perspectiva Teece (2017) realiza un aporte muy importante en la construcción del concepto 
capacidades tomando como marco teórico la economía y la estrategia, pero no considera las teorías de la organización y de la administración. Nuestro aporte tiene por objetivo llenar este vacío en la teoría. Ambos aportes permitirán construir una perspectiva integral y multidimensional que permita una comprensión completa de las organizaciones y facilitarán la realización de investigaciones teóricas, empíricas y de aplicación práctica.

CDEO es una perspectiva híbrida, ya que integra grandes campos del conocimiento que han evolucionado de manera paralela, las teorías de las organizaciones, de la administración, de la estrategia. Permitirá relacionar academias de intelectuales e investigadores en torno a temas de interés común.

Reconocemos que nuestro aporte puede ser mejorado con las críticas, comentarios y aportes de la comunidad académica. Hemos aceptado el desafío de construir teoría teniendo en mente que en México y América Latina se tiene los recursos y las capacidades para dar un aporte significativo al desarrollo del conocimiento.

En necesario profundizar el estudio en los siguientes temas: identificar las dimensiones del constructo a fin de realizar estudios empíricos; aplicar el concepto en diferentes tipos de organizaciones teniendo en cuenta su tamaño, orientando el estudio en las medianas y pequeñas empresas.

Nuestro aporte es teórico, es necesario realizar estudios empíricos teniendo en cuenta la realidad de las organizaciones mexicanas y latinoamericana, aplicando metodologías cualitativas y cuantitativas.

\section{References Références Referencias}

1. Ambrosini V, Bowman C. 2009. What are Dynamic capabilities and are they a useful construc in strategic Management? International Journal of Management Review 11 (1): 29-49.

2. Aramand M. Vallieri D. 2012. Dynamic capabilities in entrepreneurial firms: A case study approach. DOI 10.1007/s10843-012-0088-3

3. Andrews R. Beynon MJ. McDemott AM. 2016. Organizational capability in the public sector: a configuration approach. Journal of Public Administration Research and Theory: 239-258.

4. Augier M, Teece DJ. 2006. Understanding complex organization: the role of know-how, internal structure, and human behavior in the evolution of capabilities. Industrial and Corporate Change 15 (2): 395-416.

5. Barney J. 1997. Gaining and Sustainig Competitive Advantage. Addison-Wesley, MA.

6. Breznik L, Hisrich RD. 2014. Dynamic capabilities vs. innovation capability are they related? Journal of Small Business and Enterprise Development 21 (3): 368-384.
7. Braganza A. Brooks L. Nepelski D. Ali M. y Moro R. 2017. Resurce management in big data iniciatives: process and dynamic capabilities. Jornal of business Research. 70: 328-337.

8. Cohen WM, Levinthal DA. 1990. Absortive capacity: a new perspective on learning and innovation. Administrative Science quarterly 35 (1): 128-152.

9. Collis D. 1994. Research note: how valuable are organizational capabilities? Strategic Management Journal 15: 143-152.

10. Chandler AD. 1992. Organizational capabilities and the industrial enterprise. Journal of Economic Perspectives. 6 (3): 79-100.

11. Barnad Ch. 1938. The Functions of the Executive. Harvard University Press.

12. Denford JS. 2013. Building knowledge: developing a knowledge-based dynamic capabilities typology. Journal of Knowledge 17 (2): 175-194.

13. Dierickx I, Cool K. 1989. Asset stok accumulation and sustainability of competitive advantage. Management Science 35: 1504-1511.

14. Di Stefano G., Petera M, Verona G. 2010. Dynamic capabilities decostructed: a bibliographic investigation into the origins, development, and future directios of the research domin. Industrial and Corporate Change 19 (4): 1187-1204.

15. Dixon Se, Meyer KE, Day M. 2010. Stages of organizational transformation in transition economies: a dynamic capabilities approach. Journal of Managements Studies 47: 416-436.

16. Easterby-Smith A. y Prieto I.M. 2008. Dynamic capabilities and knowledge management: an integrative role for learning? British Journal of Management 1): 235-249.

17. Enkel E, Roseno A, Mezger F. 2012. Dynamic capabilities for new business creation: acrossindustry study. This paper was presented at The XXIII ISPIM Conference - Action for Innovation: Innovating from Experience - in Barcelona, Spain on 17-20 June 2012.

18. Fainshmidt S. y Frazier ML. 2016. Waht facilitates dynamic capabilities? The role of organizational climate for trust. Long Range Planning (Article in Press).

19. Foss NJ, Lyngsie J, Zahra SA. 2013. The role of external knowledge sources and organizational desingn in the process of opportunity exploration. Strategic Management Journal. 34: 1453-1471.

20. Gary MS, Wood RE. 2011. Mentals models, decision rules, and performance heterogeneidad. Strategic Management Journal. 32: 569-594.

21. Ghoshal S. Hahn M. Moran P. 1999. Management competence, firm growth and economic progress. Contribution to Political Economy 18: 121-150.

22. Gupta V. 2014. Dynamic capability delepment process, structure and behavior. Journal of International Scientific Publications 8: 1187-1203. 
23. Grant RM 1996. Toward a knowledge-based theory of the firm. Strategic Management Journal. 17: 109-122.

24. Grimaldi M, Quinto I, Rippa P. 2013. Enablig open innovation in small and medium enterprises: a dynamic capabilities approach. Knowledge and Process Management 20 (4): 199-210.

25. Hatch MJ. 1997. Organization Theory Modern Symbolic and Posmodern Perséctives. Oxford University Press: New York.

26. Helfat C. 1997. Know-how and asset complementary and Dynamic capability accumulations: the case of R5D. Strategic Management Journal 18: 393-360.

27. Helfar CE, Peteraf MA. 2015. Managerial cognitive capabilities and the microfoundations of dynamic capabilities. Strategic Management Journal. 36: 831-850.

28. Kim WC, Mauborgne R. 1997. Value innovation: the strategic logic of high growth. Harvard busines Review 75 (1): 103-112.

29. Kor Y, Mesco A. 2012. Research notes and commentaries dynamic managerial capabilities: configuration and orchestration of top executives capabilities and firms dominant logic. Strategic Management Journal 30 (13): 1375-1394.

30. Kuuluvainen A. 2012. How to concretize Dynamic capabilities? Theory and examples. Journal of Strategy and Management 5 (4): 381-392.

31. Krzakiewwicz K, Cyfert S. 2014. The strategic dimension of the dynamic capabilities of enterprises. De Gruyter 18 (2): 7-18

32. Laaksonen O, Peltoniemi M. 2016. The essence of dynamic capabilities and their measurement. International Journal of Management Reviews DOI: 10.1111/ijmr.12122

33. Lawrence PR, Lorsch JW. 1967. Differentiation and integration in complex organizations. Administrative Science Quarterly 12 (1): 1-47.

34. Luhmann N. 2012. Organización y Decisión. Herder.

35. Manning SP, Hutzschenreuter T. Strathmann. A. 2012. Emerging capability of continuous challenge? relocating knowledge work and managin process interfaces. Industrial and corporate Change, 22 (5): 1159-1193.

36. March JG. Simón HA. 1980. Teoría de la Organización. Ariel. Ciudad de México, México.

37. Mintzberg, $\mathrm{H}$. Mintzberg on Management. Free Press, United States of América.

38. Miranda JT. 2014. El Modelo de las Capacidades Dinámicas en las Organizaciones. Editorial Fenix, Mexico.

39. Pavlou P, Sawy O. 2011. Understanding the elusive black box of Dynamic capabilities. Decisión Science 42 (1): 239-273.

40. Perose ET. 1959. The Growth of the Firm. Wiley, New York.
41. Peteraf M. 1993. The cornerstones of competitive advantage: a resource-based view. Strategic Management Journal, 14 (3): 179-191.

42. Porter ME. 1987. Ventaja Competitiva Creación y Sostenimiento de un Desempeño Superior. Cecsa. Ciudad de México, México.

43. Rashidirad M, Soltari E, Salimian H. 2014. Do contextual factors matter? a missing link between competitive strategies-dynamic capabilities alignment and e-business value. Strategic Change 23: 81-92.

44. Rashidirad M, Soltari E, Syed J. 2013. Strategic alignment between competitive strategy and dynamic capability: conceptual framework and hypothesis delopment. Strategic change. 22: 213-224.

45. Ritala P. Heiman B. Humerlinna-Laukkamen P. 2016. The need for speed-unfamiliar problems, capability rigidity, and ad hoc processes in organizations. Industrial and Change 25 (5): 757-777.

46. Simon, H. El Comportamiento Administrativo Estudio de los Procesos Decisorios en la Organización Administrativa. Aguilar. Madrid España.

47. Shara S, Vredenburg H. 1998. Proactive corporate environmental strategy and the development of competitively valuable organizational capabilities. Strategic Management Journal, 19: 729-753.

48. Schilke O. 2014. On the contingent value of dynamic capabilities for competitive advantage: The nonlinear moderating effect of environmental dynamism. Strategic Management Journal 35: 179203.

49. Standler C, Helfal CE, Verona G. 2013. The impact of dynamic capabilities resource access and development. Organization Science 24 (6): 1782-1804.

50. Skokic V. Coh M. 2015. Stratec leverangin: The topline impact of dynamic capabilities. International Journal of business Administration. 6 (5): 1-15.

51. Trau F. 2017. The organizational factor and the growth of firms. Cambridge Journal of Economics. 41: 749-774.

52. Tranfield, D., Denyer, D y Smart, P. (2003). Towards a methodology for developing evidence-

53. informed management knowledge by means of systematic review. British Journal of Management, 14, 207-222.

54. Teece DJ, Pisano G, Shuen A. 1997. Dynamic capabilities and strategic management. Strategic Management Journal 18: 509-533.

55. Teece DJ. 2017. A capability theory of the firm: an economics and (strategic) management perspectives. Working Paper series $N^{\circ} 20$. Tusher Center for the Management of Intellectual Capital. 
56. Teece DJ. 2014. The foundations of enterprise performance: dynamic and ordinary capabilities in an (Economic) theory of firms. The Academy of Management Perspectives 14 (4): 328-352.

57. Tseng SM, Lee PS. 2014. The effect of knowledge management capability and dynamic capability on organizational performance. Journal of Enterprise Information Management 27 82): 158-179.

58. Tzortzaki AM. Mihiotis A. 2014. A review of knowledge management theory and future directions. Knowledge and Process Management 21 81): 29-41.

59. Ulrich D. Lake D. 1991. Organizational capability: creating competitive advantage. Academy of Management Executive 5 (1): 77-92.

60. Vivas S. 2005. Competitive advantage and strategy formulation: the key role of Dynamic capabilities. Management Decision 45 (5/6): 661-669.

61. Vogel R, Guttel WH. 2013. The dynamic capability view in strategic management: a bibliometric review. International Journal of Management Reviews 15: 426-446.

62. Volberda HW, Weerdt N, Verwaal E, Stienstra M, Verdu AJ. 2012. Contingency fit, institutional fit, and firm performance-environment relationships. Organization Science 23 (4): 1040-1054.

63. Wang Y. 2016. Environmental dynamism, trust dynamic capabilities of family businesses. International Journal of Entrepreneurial Behavior \& Research 22 (5): 7

64. Winter SG. 2017. Pursuing the evolucionary agenda in economic and management research. Cambridge Journal of economics. 41: 721-747.

65. Winter SG. 2003. Understanding Dynamic capabilities. Strategic Management Journal 24: 991-995.

66. Zahra S, George G. 2002. Absortive capacity. A review, reconceptualization and extension. Academy of Management Review 27 (2): 213-240.

67. Zheng S., Zhang W., Wu X. y Du J. Know ledgebased Dynamic capabilities and innovation in networked enviroments. Journal of Knowledge Management 15 (6): 1035-1051. 